\title{
Beyond Adaptive Sports: Challenges \& Opportunities to Improve Accessibility and Analytics
}

\author{
Rushil Khurana \\ rushil@cmu.edu \\ Carnegie Mellon University \\ Pittsburgh, PA
}

\author{
Ashley Wang \\ awwang@andrew.cmu.edu \\ Carnegie Mellon University \\ Pittsburgh, PA
}

\author{
Patrick Carrington \\ pcarrington@cmu.edu \\ Carnegie Mellon University \\ Pittsburgh, PA
}

\begin{abstract}
A recent surge in sensing platforms for sports has been accompanied by drastic improvements in the quality of data analytics. This improved quality has catalyzed notable progress in training techniques, athletic performance tracking, real-time strategy management, and even better refereeing. However, despite a sustained growth in the number of para-athletes, there has been little exploration into the accessibility and data analytics needs for adaptive sports. We interviewed 18 participants in different roles (athletes, coaches, and high-performance managers) across six adaptive sports. We probed them on their current practices, existing challenges, and analytical needs. We uncovered common themes prevalent across all six sports and further examined findings in three groups: (1) blind sports; (2) wheelchair sports; and (3) adaptive sports with high equipment. Our study highlights the challenges faced by different adaptive sports and unearths opportunities for future research to improve accessibility and address specific needs for each sport.
\end{abstract}

\section{CCS CONCEPTS}

- Human-centered computing $\rightarrow$ Accessibility technologies.

\section{KEYWORDS}

adaptive sports, accessibility, analytics

\section{ACM Reference Format:}

Rushil Khurana, Ashley Wang, and Patrick Carrington. 2021. Beyond Adaptive Sports: Challenges \& Opportunities to Improve Accessibility and Analytics. In The 23rd International ACM SIGACCESS Conference on Computers and Accessibility (ASSETS '21), October 18-22, 2021, Virtual Event, USA. ACM, New York, NY, USA, 11 pages. https://doi.org/10.1145/3441852.3471223

\section{INTRODUCTION}

From coaching to strategy, training to in-game adjustments and refereeing to viewer experience-the use of data analytics has revolutionized every aspect of sports. The data for sports analytics is typically sourced from user-worn smartwatches and trackers, environmental sensors such as video cameras [13, 16, 27] and, in some cases, sensor-laden equipment $[34,55]$. The global market size of the sports analytics industry was estimated to be $\$ 1.9$ billion

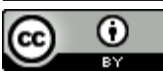

This work is licensed under a Creative Commons Attribution International 4.0 License.

ASSETS '21, October 18-22, 2021, Virtual Event, USA

(c) 2021 Copyright held by the owner/author(s).

ACM ISBN 978-1-4503-8306-6/21/10.

https://doi.org/10.1145/3441852.3471223 in $2019^{1}$. However, despite this incredible growth and use of technology to gather analytics in able-bodied sports, there has been little support for their counterpart, adaptive sports.

The first organized Paralympic games date occurred in 1948, and were officially recognized as the first summer Paralympic games in 1960. The Paralympic community has strived for equality and celebration of excellence in adaptive sports. Besides the physical benefits associated with staying active and participating in sports, adaptive sports in particular also act as a support system within the community. For the athletes, sports provide a sense of belonging and provide opportunities to learn from their peers and boost their confidence [32]. In fact, it has also been shown that participation in sports positively impacts the likelihood of employment for people with disabilities [31]. There is a significant improvement in the quality of life for athletes who participate in adaptive sports.

Despite the physical and mental benefits of adaptive sports, there has been a lack of technological innovation that caters to this community. Many technological solutions are modeled for the able-bodied sports and do not cater to the specific needs of their adaptive counterpart. Something as simple as counting the steps and measuring the distance covered by an athlete is designed for an able-bodied athlete; the step counter algorithm in fitness trackers is unable to measure the movement or the push of a wheelchair, making one of the most basic statistics inaccessible for para-athletes [1]. In fact, wheelchair users also have limited trust in the step counts shown on fitness trackers [7].

To understand the challenges and opportunities to improve the accessibility of adaptive sports and gather analytics, we conducted a series of semi-structured interviews with 18 individuals in different roles (program managers, coaches, athletes and analysts) associated with six different adaptive sports (wheelchair basketball, wheelchair rugby, wheelchair tennis, para powerlifting, blind hockey and blind baseball). The goal of our work is to uncover practical opportunities for researchers and technologists to address the needs specific to the adaptive counterpart of each sport. We first analyzed needs that were common across all sports and provide an overview of accessibility needs. Next, we analyzed our findings in three groups: (1) blind sports; (2) wheelchair sports; and (3) adaptive sports with high equipment. Some key findings from our process are:

(1) Blind sports are still trying to improve the accessibility of their basic game mechanics. For example, blind hockey uses a sound sensor embedded into the puck. However, these pucks are not durable and do not last for more than a couple of games.

(2) Competitive wheelchairs are customized for each individual athlete and cost several thousand dollars. The chair setup

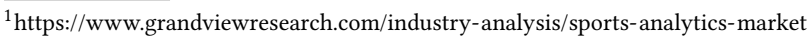


impacts athlete comfort and their performance. The process to determine an appropriate setup is ad-hoc and there are no concrete guidelines or tests to do so.

(3) Adaptations of sports to make them accessible include wheelchairs or technology imbued into otherwise ordinary objects such as a sound-emitting puck for blind hockey. As such, equipment instrumentation is rife with opportunities to cater to the technological and analytical needs of a sport. For example, a sensor embedded in a barbell for a para powerlifter can aid the athlete in training, provide the judges with a visualization of the accurate trajectory and provide fans with additional information about an athlete's performance (bar movement and velocity) and improve the viewing experience.

We make two contributions in this paper. First, we outline technological needs and challenges faced by individuals associated with different adaptive sports. We outline key differences based on the kind of disability, and the amount of equipment used in the sport itself. Secondly, our findings reveal opportunities to make a sport more accessible and aid different stakeholders based on their particular needs.

\section{RELATED WORK}

Sports technology spans across numerous applications including augmentation tools to assist athletes, training aids, arming referees with instant replays and slow-motion videos enhancing how stakeholders engage with a sport. In this section, we first survey sports technologies to provide an overview of how technology has improved the user experience of athletes, coaches and umpires.

Data analytics is the backbone of modern-day sports. In the next section we specifically highlight how technology has improved the data collection and inference for sports. We summarize both commercial and research efforts to sense, track and capture data analytics in different sports.

Finally, we review prior work at the intersection of technology and adaptive sports. We provide a background of how technology (or lack thereof) has impacted the accessibility of a sport, existing solutions to improve the experience of para-athletes, and the state of data analytics in adaptive sports.

\subsection{Sports Technology To Improve User Experience}

In this section, we examine examples of how technological innovations in sports has impacted how different stakeholders are engaged and supported in their tasks.

The most common use of technology has been to develop training aids that can provide immediate feedback to the user. For example, augmented climbing uses a combination of projected graphics and a body suit combined with a depth camera. It tracks the position of climbers to assist them in finding appropriate routes and develop their motor skills [23]. Similarly, Han et al. built an augmented reality application to assist individuals in learning Tai-Chi Chuan [20]. The system comprises of virtual coaches that assist the user in learning new moves, a drone that uses the onboard camera to capture the user's posture and pose, and an augmented mirror that displays this feedback.
Prior work has also looked at using on-body sensors such as IMUs and pressure sensors to infer movements. ClimbingAssist uses pressure sensitive foil on the insole of a climber's shoe to provide feedback depending on the pressure on the foot and movement speed [15]. On the other hand, Daniel Spelmezan mounted a snowboard with four pressure sensors to measure the difference in pressure in toes versus heels to identify a riding edge in snowboarding [47]. Such a system allows the coach to correct the athlete in real-time rather than post-session analysis. Similar training systems exists for badminton [40], baseball [30] and basketball [38].

There have been improvements in the kind of feedback received by the athlete as well. FootStriker is a wearable running assistant that uses force sensing resistors to detect heel strike, and an EMS device to actuate the calf muscles to manage the foot angle before the foot lands on the ground [21]. In fact, there have even been studies to understand what feedback modality would be best suited for an athlete. Rob Gray investigated how different feedback modalities (audio, visual and tactile) are used by a baseball player to determine swing accuracy [18]. Another facet of training is athlete conditioning. There has been prior work in tracking exercises [24] using cameras or wrist-worn devices [36]. Beyond sensing, on-body devices have also been used to build animated 3D visualizations while a person exercises for improved feedback [45].

Sports technology to improve user experience is not limited to training. It is also used to improve-in game experience. JackIn Airsoft uses wearable cameras to generate a map that aids strategizing in Airsoft [27]. It borrows the idea of using maps in video games for in-person Airsoft. Other in-game improvements include systems such as SkiAR: a collaborative wearable AR system for skiers to share pictures, prior routes and encountered hazards on a shared map [14]. This information can be used by others to prevent injuries on dangerous routes, or guidance if needed. Prior work has also examined opportunities to build future in-game experiences. Colley et al. developed a virtual reality immersion where skiers wear a head mounted display as they go down a beginner slope [12] and experience an alternative reality through the HMD in what they term as blended reality.

Umpires and sports broadcasters are also key stakeholders in sports. And as such, Hawk Eye system is an integral data capturing system for tennis that uses cameras to track the ball motion [39]. It is used both for umpiring decisions and sports broadcasting. Similarly, FIFA uses goal-line technology to assist referees in determining if a goal was scored. Researchers have also developed tools to assist judges in scoring mixed martial arts matches by determining impact of kicks and punches [11]. Similar tools are used for sports broadcasting as well. Ekin et al. built a system to automatically summarize a football game to extract key moments such as all the goals scored, and slow-motion videos of those moments [13]. It is useful for sport broadcasters to quickly process videos and share relevant segments. And lastly, there exists prior work to improve the engagement of a live audience using wearable inertial sensors to track clapping and cheering to be used as a voting system [51]. While such a system does not produce objective results, it aims to improve spectator experience by giving the audience a voice in the voting process. 


\subsection{Sports Technology for Data Analytics}

Nowadays, sports rely heavily on data analytics to make data-driven decisions. Sports tech is used to capture quantifiable measures that represents an athlete's (or a team's) performance. These analytics are then used to change training practices, in-game strategies, and even as selection criteria for teams.

A lot of the data analytics are captured during training sessions to help athletes focus on their weaknesses. For example, Wang et al. used 4 inertial sensors placed on each wrist, the torso and the right ankle to recognize and classify 14 different badminton strokes with $98 \%$ accuracy [55]. A post-game analysis can aid athletes in determining if they used the right stroke, their speed and accuracy. Similarly, Maglott et al. built an arm sleeve with two IMUs to capture the upper arm and forearm kinematics [34] for basketball athletes. Their setup is able to identify key differences in arm motion timing, a critical characteristic to train and improve shooter accuracy. Similar work to passively track relevant analytics has been done in other sports such as swimming [5,33], roller derby [49] and soccer [16, 19].

There are several commercial efforts to capture these analytics as well. For instance, Major League Baseball uses StatCast [9], a camera and radar-based system to capture and infer analytics such as running speed, pitching, swing trajectory and more. The data from StatCast has readily been used to infer analytics and build dashboards [29]. Similarly, NBA employs Second Spectrum ${ }^{2}$, a camera-based system that uses machine learning to generate insights from the gathered analytics (e.g., number of passes and angle of shot). There are more generalizable solutions such as PlayerTek ${ }^{3}$, developed to track both individual and team performance. It has an IMU, and GPS unit as well to track player positions to derive analytics such as movement patterns.

In some cases, software solutions built on top of these analytics provide direct feedback to the participants. For example, virtual coaches [10] help users train for marathons by suggesting adjustments to running speed in real-time. It helps beginners maintain a consistent training intensity. Now that data tracking systems in sports are generating large amounts of data, developers can create intelligent systems and interfaces to derive relevant analytics from big sports data [46].

There are two key takeaways from this summary. First, there has been a deep positive impact of technology in tracking athletes, capturing informative signals and inferring insights from data analytics. Secondly, the data tracking systems typically rely on either video-based sensing or user instrumentation such as wrist-worn sensors. However, the on-body sensing systems tend to be used for training purposes only and not for in-game data collection. We hypothesize that this may be to prevent any interference or injuries in the game. There is also a noticeable lack of equipment instrumentation to gather analytics. Similar to on-body based devices, smart equipment that do exist are developed for training purposes only $[2,28,44,49]$.

\footnotetext{
${ }^{2}$ https://www.secondspectrum.com/

${ }^{3}$ https://www.playertek.com/
}

\subsection{Adaptive Sports Technology}

In this section we examine how prior work has used technology for enhancing adaptive sports. This is perhaps the most closely related to our work. A key use of technology has been to improve the accessibility of a sport for visually impaired athletes. Goby uses a camera worn on a visually impaired swimmer's thigh to detect if they are nearing a wall or going out of the swimming lane and notifies them via an audio notification [37]. Similar navigational information is conveyed to visually impaired athletes using IMUs. Aggravi et al. put two vibrating bracelets on the forearms of a visually impaired skier to provide them directional information using haptic feedback [3]. On the other hand, Folmer takes an environmental-sensing approach and used aerial-robots to guide blind runners on a path using audio cues [17]. Similarly, Eyes-Free Yoga uses a camera to track a user's posture and provide them feedback on their yoga poses [41].

There have been some concentrated efforts to instrument the wheelchair to gather in-game analytics. Carrington et al. used an IMU attached to a wheelchair to capture motion-based analytics for wheelchair basketball athletes [8]. It builds upon prior systems that capture similar analytics using IMUs [22, 52], indoor RF positioning systems [35] and reed switches [48, 50]. 3D force sensors have also been used in the past to measure force applied to handcycle handgrips [53] and have been combined with motion analysis to catalog athletic performance for handcycling and wheelchair racing [4].

Technology in able-bodied sports plays an important role in not only improving the user experience, but also capturing granular analytics that aid all stakeholders in the sport. While some of those technologies can be retrofitted for use in adaptive sports, there is a significant gap in research and development of platforms that cater specifically to the needs of adaptive sports. There are no widely accepted commercial technologies for adaptive sports.

There are only a handful of systems designed to improve accessibility, and fewer to gather analytics, impeding adaptive sports from the same data-driven decision-making process taken by ablebodied sports. The goal of our study is to help technologists and researchers identify opportunities and key areas to contribute such that stakeholders in adaptive sports are equally empowered as their able-bodied counterparts.

\section{METHODS}

\subsection{Pilot}

We first developed a list of around eight questions to elicit challenges and needs in adaptive sports. Each sport is governed by a different set of rules and regulations, uses different equipment, and is likely to have a mix of both overlapping and unique challenges with other adaptive sports. These questions were used as probes in our semi-structured interview process. We ran two pilot interviews with an athlete and a coach in adaptive sports. We enquired about participants current practices and use of technology. Next, we asked about their technological and analytical needs, and any other issues pertaining to the sport. The interviewees discussed issues with accessibility, and lack of resources, confirming our speculation that adaptive sports are far behind their able-bodied counterpart in sports analytics. 


\subsection{Study Procedure}

We conducted 18 semi-structured interviews to understand the challenges and opportunities surrounding improving the accessibility of adaptive sports and gathering analytics. The interviews were conducted remotely, leveraging video or audio conferencing. Each interview lasted 45 minutes on average. Based on our pilot interviews, we added three new probes to our interview protocol. The major themes of our questions were: (1) current practices and technology; (2) technological and analytical needs; (3) athlete tendencies and anecdotal data; and (4) role of the equipment in their respective sport. We used these probes but dove deeper into certain topics when required. Other questions were tailored specifically to each different sport.

\subsection{Participants}

We used snowball sampling to recruit interviewees. We tried to balance interviewees in terms of the role they play in a particular sport (manager, coach, athlete, analyst) within each sport. We recruited participants from six different adaptive sports. We also tried to capture representation from sports with different kinds of disability (see Table 1). We found it hard to quantify and compare years of experience, but we had a mix of participants including coaches/athletes who only play in leagues, athletes who play competitively at the national level, athletes who have been playing for only 3-4 years, and coaches with over 25 years of experience.

\subsection{Data Collection and Analysis}

All interviews were audio recorded and transcribed. We performed open coding on the transcribed interviews to identify major themes in the data. We followed a collaborative coding process [42]. Multiple members of the research team were involved in the coding process and reached a consensus for codes generated through this process. We used thematic analysis [6] on our codes in three different subsets: (1) blind sports; (2) wheelchair-based sports; and (3) adaptive sports with high equipment. We identify challenges and opportunities to improve accessibility and gather analytics in each group.

\section{FINDINGS}

First, We discuss major challenges that span across all sports in our study. We analyzed interview data from all participants (regardless of sports) together. These overarching challenges exacerbate other issues outlined in the latter sections for different categories of sports, and thus are critical to outline before we discuss each group individually.

Next, we look at smaller categories to better understand the needs across different dimensions. First, we examine if a different kind of disability (visual impairment vs paraplegia) impacts the accessibility of a sport. Then, in our next category we combine all sports that rely on athletes using a wheelchair. Our last category looks at sports with high equipment (i.e., the sport requires a lot of equipment; for example, blind hockey) needs to explore if the amount of equipment needed to play an adaptive sport opens up any new opportunities. For each group, we focus on themes that were corroborated by a significant discussion by multiple participants.

\subsection{Common Issues in Adaptive Sports}

The initial lens for our research was that of how analytics tech could transform the experiences of participants. However, our study revealed that investing in the organizational needs of sustaining the sport and program takes precedence over analytics tech at this stage. In fact, technologies that enable playing some sports are still underdeveloped. Therefore, before we discuss challenges and opportunities with different sports, we first want to highlight challenges that span across all adaptive sports.

We want to stress that these are challenges faced by professionallevel athletes in adaptive sports. While some of the financial and resource constraints would also be obstacles at amateur levels of an able-bodied sports, these are challenges faced by adaptive sport athletes even at the highest levels. All of our participants are engaged in their respective sports in support of national or state-level competitive teams.

4.1.1 Financial Limitations and Cost. The biggest hurdle in any adaptive sport is its resource and financial constraints. More than half of the adaptive sports were underfunded and struggled to obtain resources such as permanent staff members. They typically rely on people who are passionate about the sport and volunteer their time outside of their primary job. This volunteer base leads to a shortage of coaches and staff that can constantly cater to the needs of the sports organization. Even when a volunteer leaves and another steps in, the knowledge transfer and acclimation period slows down the development of athletes. One wheelchair rugby coach/high performance manager describes the difficulties of having few permanent staff members.

"Um, well, one thing is, uh, staffing. Like we have a very, we have a very small staff of 10, um, and that 10 may or may not be there at any given time, um, because all work full time [at other jobs]; the only full-time employees on the staff are myself, and then also our head coach." [HPM-2]

Some sports organizations did not have access to proper playing fields. One participant [A-6] in beepball explained that there was little to no support for the blind teams to have access to a well-maintained field. In more than half of the adaptive sports, the athletes paid for their own travel and accommodations when competing in tournaments. For wheelchair sports, the wheelchairs are customized for each athlete depending on their disability and comfort. Each chair costs several thousand dollars which creates a bottleneck for individuals to join the sport. These resource and financial constraints impede the ability of adaptive sports to grow, gain popularity, and attract more people to participate. The lack of popularity and support structure leads to limited resources for novices to get involved in the sport, locally. One participant describes the challenge of participating in local programs.

"For instance, for myself, I could go play an adult pickup basketball league all over the place, you know, there's, there's those leagues everywhere. [But] if I want to play on a wheelchair basketball team, for instance. I' $m$ down here in Florida, the closest team for me right now that I'm aware of is three hours away. You know, so that's a lot of travel and a lot of costs associated with being part of that program." [HPM-1] 
Table 1: Study participants by Sport and Role

\begin{tabular}{|l|l|l|}
\hline Sport & Number of Participants & Participant Roles [Participant ID] \\
\hline Wheelchair Basketball & 3 & High-Performance Manager [HPM-1], Coach [C-1], Athlete [A-1] \\
\hline Wheelchair Rugby & 3 & High-Performance Manager [HPM-2], Coach [C-2], Athlete [A-2] \\
\hline Wheelchair Tennis & 3 & High-Performance Manager [HPM-3], Coach [C-3], Data Analyst [DA-1] \\
\hline Para Powerlifting & 3 & High-Performance Manager [HPM-4], Coach [C-4], Athlete [A-3] \\
\hline Blind Hockey & 3 & General Manager/Coach [C-5], Athlete [A-4], Athlete [A-5] \\
\hline Beepball (Blind Baseball) & 2 & Coach [C-6], Athlete [A-6] \\
\hline Manufacturer & 1 & Wheelchair Manufacturer [WM-1] \\
\hline
\end{tabular}

Adaptive sports are often community built and driven, thus there is a subtle but important shift in incentives from elite-programs driving interest to individuals forming programs. Thus, the scarcity of existing local programs makes it difficult for individual athletes to create the communities that drive the sport by traditional means.

Additionally, financial constraints also limit the frequency of training camps conducted by the sports organizations. Only a couple of national teams reported regular training camps whereas other organizations, including some national teams, reported that they are only physically able to meet and train 4-5 times a year for a few days at a time. A sport such as para-powerlifting is able to use video-conferencing tools for remote coaching, but that introduces its own set of challenges (discussed in a later section). If the athletes cannot find resources to train or play casual games outside of these training camps, then it limits their athletic potential and impacts the whole sport negatively.

4.1.2 Lack of adaptive sports data. For technology to aid analytics, a big organizational challenge across all sports is lack of data pertaining to adaptive sports. There have been dozens of studies, composed of years of research into understanding different aspects of a sport and building technology to support its needs. However, this research and development process typically focuses on able-bodied sports and does not take into account specific issues faced in adaptive sports. We speculated that the lack of such data is a challenge and it was corroborated by our interviewees. The participants discussed the inability of current fitness trackers to accurately track activities for people with disabilities. One para-powerlifting athlete explains how his training lacks tracking using data.

"Currently, there is no tracking via technology. Um, it's mostly me and my coaches staying in tuned with my body and with my training currently." [A-3]

When athletes cannot even track something as simple as the number of calories burnt, it calls into question the accessibility of these technologies. Prior research has shown that being able to visualize and reflect on personal analytics positively impacts athletes and improves their mental and physical well-being [43] This bias in tracking solutions creates a barrier for para-athletes to improve their overall well-being and performance.

4.1.3 Reliance on Video. Lastly, the existing technology used by all sports relies heavily on videos (e.g., DartFish). These videos are recorded, tagged and analyzed to gather the analytics needed by coaches and athletes to review their performance. A wheelchair tennis high performance manager describes this process.

"They have a large coaching staff that brings video cameras and mounting devices with them, and they're able to get those matches recorded. And then from there, they have an analytics team that they download the videos and send to, and then they tag those matches." [HPM-3]

However, video solutions are a necessity for able-bodied sports because it is hard to put devices on an athlete's body without risk of it breaking or causing injuries to others. Even though video-based technology can be used in adaptive sports, there are two big issues. First, fully automated video analytics tools use kinematic models derived from non-adaptive sports that do not translate for adaptations like wheelchair sports; and secondly the semi-automated ones require video taggers (resources) that adaptive sports organizations don't usually have.

Our participants still use these tools but with limited manpower and time; the amount of data they are able to collect/tag is sparse in comparison to their non-adaptive counterparts. Unfortunately, due to the lack of tools to support adaptive sports analytics, these teams have to rely on the popular choice in the non-adaptive counterpart and repurpose it for their own use. But even when the appropriate technology is available, reliance on video technology for tracking purposes combined with the lack of proper infrastructure can still lead to challenges, as a wheelchair tennis high performance manager explains.

"Um, it's a challenge for us in that most tournaments don't have the video technology (from an infrastructure standpoint) to video record every match that our professional athletes play. It's very easy at the Grand Slam tournaments, you know, cause those events all have, they have video coverage on every court." [HPM-3]

\subsection{Group 1: Blind Sports}

We first looked at both blind sports together (blind hockey, beepball) and compared it with other adaptive sports. We noticed that there was a striking lack of analytic needs from either of the blind sport. They were not using much technology to track and improve the athlete's performance, nor did they express it as a major challenge. However, it does not mean that blind sports do not value or require sports analytics. Our interviews revealed that the primary focus of all stakeholders involved in blind sports was to improve the 
game mechanics to ensure that the game itself can be made more accessible in the way it is played, and the equipment it uses.

The lack of resources in blind sports include leveled playing fields for beep ball, ice time on rinks for blind hockey, lack of paid employees leading to unorganized pick-up games, limited volunteers and much more. It not only impacts the organization of sport, but also decreases the visibility and outreach of the sport.

Despite these financial and resource constraints, there are communities of athletes that work hard and love to play the adaptive sport. Based on our interviews we discovered three major themes that can aid and improve the experience and accessibility of blind sports. We discuss each theme in detail.

4.2.1 Theme 1: Lack of Robust Adaptive Equipment. A unique addition to blind sports is the use of technology, particularly sound sensors to make them adaptive. For example, the puck in blind hockey is hollow with a ball bearing inside that makes a noise so that the athletes can track it. However, this additional design constraint introduces a new challenge-equipment robustness. It was quite evident in our interviews that this is still an open challenge. All hockey participants described that the adaptive pucks do not last for more than a couple of games as opposed to a regular puck that can last years. One athlete described the state of the puck as follows:

It's made of metal and has ball bearings inside of it, which is extremely weird. And when you have metal on metal, when that puck hits the post, it just, it dents. [...] The joke is what type of polyhedron is that puck. Because literally, in an hour, it gets destroyed. [A-5]

The participants discussed several flaws in the current design of an adaptive puck. The inside of a new puck is smooth, so the ball bearings initially do not make much noise. It makes it harder for someone with very low vision to track the puck. As the game progresses and the puck gets some dents, it becomes louder and makes it easier to track. It only makes a noise when the puck is moving, but it stops making noise when it is completely still. While this particular design works 'well enough' for a low vision athlete, the goalies in blind hockey are typically totally blind and this flawed design of the puck prevents them from tracking the puck at all times, making the game partly inaccessible. One of our participants who was also an avid blind soccer player described similar challenges with the adaptive soccer ball where the sound dampens on grass making it challenging for low vision athletes to track the ball. This highlights a common challenge in the approach towards the design of sound-based adaptive sports equipment. They are neither comprehensive nor robust enough to function at all times.

Our work is not the first one to notice this issue. There have been past explorations to create prototype audio pucks designed to improve accessibility. However, these ideas require fabrication (injection moulding) that is expensive. Without a high volume demand, this manufacturing is not practically feasible. The scarcity of programs diminishes demand, which raises manufacturing costs [25]; but financial constraints prohibit use of expensive technology making it a vicious cycle preventing any improvements in the sport.

Similar issues have been described with the equipment used in beep ball. The bases that make sounds are wired. When someone steps on the wire, it gets damaged and needs repair or if it unplugs, the game has to pause until the base is fixed. Also, the ball used in beep ball is a modified softball with a sound sensor. It is also prone to breaking down much like the adaptive puck in hockey.

Moreover, any sport that relies heavily on sound runs into the trouble of the useful sounds getting drowned in noise. And as such, beep baseball is played with a quiet crowd to not interfere with the game. In sports with indoor arenas such as blind hockey, the echo and reverberations are different depending on the arena dimensions.

4.2.2 Theme 2: Need for Accessible Training Aids. Our participants voiced a need for suitable training aids to introduce novices to the sport. The necessary skills required to play a certain sport are hard to learn for a blind person.

Our interviews revealed that the training aids developed for ablebodied sports rely on visual cues. It is hard for blind participants to use the same tools to learn the game and presents a need to build accessible aids to learn basic skills in a sport. For example, to teach ice skating/balancing, coaches have to use makeshift aids such as a hollow wiffle ball, retrofitted with bells and attached to the skates. The audio cues are used by novices to perfect their balance/skating. Another challenge that emerged was the difficulty for multiple players to practice dribbling the puck at the same time. When multiple sound-emitting pucks are in close vicinity, it causes confusion and disorientation for players.

This challenge of accessibility is not unique to learning a new skill, but also impacts developing strategies for games. The whiteboard/game tape paradigm used in able-bodied sports is inaccessible by visually impaired athletes. To overcome this issue, hockey coaches have started using a tactile representation of the ice rink using raised wooden sticks. However, this method is currently primitive and limited in the amount of information it can convey to the athletes. It does not allow for quick modifications, and requires effort to build different boards for each new strategy. This approach to imbue tactile cues is useful, but not scalable.

He might show where he wants everyone to line up and then he'll put Xs, and, or he's doing a play at a timeout. He'll tell his players where to position. It's a visual thing. Well, we can't use that. But what we've done is take that whiteboard and wood sticks and raised like the outline of the rink is in blue. [C-5]

In contrast, other adaptive sports (e.g. wheelchair sports) do not face the same challenges in training and strategy. Even though there are several adaptations needed to make a sport accessible, the training and strategy aids employed by able-bodied sports can be used by coaches and athletes in those cases. For example, a wheelchair basketball athlete that learned to play standup basketball was able to use the same skills, and only needed to learn the chair skills needed to play the adaptive counterpart.

[He had] never heard of wheelchair basketball, but he was a single amputee. He had lived with his disability, his entire life. He had played standup basketball and didn't know wheelchair basketball existed. His transition to the sport was pretty quick and he went from, um, you know, the collegiate, the collegiate level to playing in some international competitions within, within like a 4-5-year time 
span. But he had that basketball knowledge that that really helped progress him. And then the chair skills was the only piece that he had to improve upon. [C-1]

4.2.3 Theme 3: Need for Improved Communication Tools. A common theme in the challenges and needs shared by our blind athletes was improvement in communication. Communication is a key asset in any sport (adaptive or otherwise) but it is of paramount importance for visually impaired athletes. It spans across all aspects of the game. A visually-impaired athlete in training needs instructions that do not rely on visual cues. During the game, a common challenge in blind sports adaptation is to convey the 'state of gameplay' to each athlete. In fact, certain sports require clear communication as a part of the adaptive game. In contact sports such as soccer, the athletes have to announce "I'm coming", and in beep baseball, there are sighted spotters on the sideline guiding the blind athletes as a part of the game. It makes the adaptation reliant on the presence of a sighted person. In our interviews, while the coaches and managers focused on the need for communication tools as training aids or to prevent injuries, the athletes pointed out their use to improve the accessibility of the game.

This need for improved communication is not limited to just between athletes. Athletes also expressed interest in how the environment conveys information to them. For example, one hockey athlete suggested using beacons on the goalpost to assist them in localizing its position, which in turn allows them to practice their shots on an empty net. This simple addition of a sensor in the environment to localize the position of an empty net helps a visually-impaired individual to train without assistance from another person. One hockey athlete describes how he has developed his own way of implementing audio cues in his solo training.

"So there's tricks you can do for if I'm going to practice them all myself, but I'm trying to block a location. I could have my, somebody run around me on the string, if you will. And then I just put a beacon back there. It could be any noise maker, whatever it is, the phone radio, whatever. And this way I'm getting in a position to block the ankle, but I'm using that noisemaker as my orientation point, at least with a goal. And then from the goal, I can tell if I'm hearing sound from that direction, I can tell you what direction I am facing. Am I turned 30 degrees in the wrong direction? Because the audible clues is then I can reorient myself to that orientation point." [A-4]

There is a gap in the tools and technology used to improve this communication process in making a sport more accessible to blind athletes.

\subsection{Group 2: Wheelchair-Based Sports}

Next we looked at sports where the athletes use a wheelchair (wheelchair basketball, wheelchair rugby, and wheelchair tennis). We uncovered two major themes which we discuss below.

4.3.1 Theme 1: Tracking Athlete Conditioning and Performance. Wheelchair sports typically rely on either video-based technology (e.g., DartFish) or laser timers to measure statistics such as distance covered, top speed of an athlete, and their acceleration. A typical national team selection process or training camp in wheelchair-based sports relies on evaluating sprints, measuring and assessing athletes on core criteria such as push mechanic, speed and agility. A cumulative picture of these analytics allows coaches to better gauge an athlete's physical conditioning. Further, the highperformance manager and analyst in wheelchair tennis expressed a specific interest in measuring the physical load on an athlete's body. They described that they can accurately estimate the physical load exerted on an athlete's body by tracking and cumulatively analyzing factors such as amount of acceleration/deceleration, stoppage time, turning time and total time spent on the court during a session.

Besides athlete conditioning, participants from wheelchair sports also expressed their need to track their tendencies. The participants want to know the tendencies of an athlete such as if they rely on their left or right side to push more, the position they grab the wheel, if they like to lean forward or backward, and measure its impact on their performance. Such analytics help coaches and athletes make an informed decision on how to adjust their training to improve performance. The current best practices taught as a part of most adaptive sports are rooted in anecdotal data. A quote from a wheelchair basketball athlete describes one such data point:

"The idea is that you should be able to grab your wheel at like a 11 or 10 o'clock position. And then push over the top and through, and that's the fastest way to get going. But if you are a more severely disabled person, grabbing your wheel at the 12 o'clock position is as far back as you should ever go. Any further back and you start to sacrifice acceleration. And so that changes how people are taught pushing based off of their disability. Um, so, and that's all anecdotal." [A-1]

The ability to capture and visualize granular data, understand these tendencies, and validate or disapprove these anecdotal beliefs would better inform an athlete's training and enhance their performance. It also helps build up a dataset that can be leveraged across different wheelchair-based sports to analyze the movement mechanics of a wheelchair that are shared across all sports. Such a dataset can then be used to build better algorithms for tracking solutions, a problem seen across all adaptive sports.

4.3.2 Theme 2: Wheelchair Customization, Setup and Maintenance. The second major theme that emerged from our interviews centered around the customization of the wheelchair. Each wheelchair used by an athlete is custom designed based on their disability, kind of injury and their size. By virtue of their design, they are customized to suit a particular person. However, they are also expensive. This introduces a barrier to even enter the sport. One participant remarked:

"You're going to get a custom fit chair built for you. But, there's a range, you know? I mean, if I had to give you an overall range, I would say you could spend any where from $\$ 2,000$ up to $\$ 10,000$, you know?" [HPM-1] 
The wheelchair customization is based on an athlete's disability and how it impacts their comfort in the wheelchair. While certain customizations and chair setups are straightforward based on the injury, there are other aspects of a chair profile that may require a more careful assessment.

"Um, but what's the right equipment? What's the right chair profile? Do you need to have a certain amount of dump based on your spinal cord injury? Are you going to have ergonomic seating versus standard, you know, dumped seating? Uh, we're going to have a recline on the backrest to accommodate. You know, scoliosis or a decline on the backrest because of, you know, hyper hypermobility in your lower back." [WM-1]

However, there is a lack of a data-driven process to capture what settings are optimal for a particular athlete. The process of customizing the chair for each athlete relies on the expert being able to recognize patterns they may have seen before. This approach is non-analytical and may even lead to a monopoly of a handful of experts in the wheelchair manufacturing business.

Once the chair is customized to an athlete's liking, the maintenance and upkeep of the wheelchair also costs money. The physical nature of the sport, especially in games like wheelchair rugby, impact the longevity of the wheelchair. The wear and tear leads to issues that make the chair uncomfortable, negatively impact the athlete's performance and in some case cause injuries. As such, there are personnel who assist the Paralympic teams with preparations and maintenance of the wheelchair. It includes checking up the integrity of the nuts and bolts, lubricating parts, maximizing air pressures and more. Beyond the initial customization of the wheelchair, there is a lot of effort involved in maintaining the quality of the wheelchair at a competitive level.

Besides catering to an athlete's specific disability and injury, there are setup customizations that may happen at a later stage to alter an athlete's performance. Key parameters such as wheel width, angle of tilt, and height of the chair affect an athlete's performance and custom setups may be required for different athletes. One participant summarized this challenge as:

"If you're not in the right chair setup, it can affect your speed, it can affect your push, it can affect, uh, I mean it can affect everything if it's not the right fit or chair setup." [HPM-2]

The participants also stated that they have specific training goals and the wheelchair setup is a big component in helping them achieve those goals. Currently the coach or the wheelchair manufacturer that provides support may assess if some customizations will help improve an aspect of their game. Based on this assessment, the wheelchair setup is modified to align with the athlete's goal and improve their performance. For example, an athlete may prefer to sacrifice their top speed for better acceleration based on their playing style and their role in the game. However, this process currently happens in an ad-hoc manner without much underlying data to support the decision-making process.

Lastly, we wanted to highlight that wheelchair sports exhibit specific injuries due to the use of a wheelchair in the sport. The physical load exerted on an athlete's body due to continually pushing the wheelchair during games leads to overuse of shoulders and rotator cuff injuries. One participant even pointed out that chair setup plays a vital role in reducing the chances of such injuries.

\subsection{Group 3: Adaptive Sports with High Equipment}

Based on the responses of our probe on the role of equipment combined with our findings from the last two groups, we determined that we should also examine a grouping of adaptive sports with high equipment. So, in this grouping we look at wheelchair tennis, blind hockey, beepball and para powerlifting. These sports tend to have more equipment compared to the other sports and each athlete has ownership of that equipment i.e., they are not shared during gameplay.

Among these, para powerlifting is unique in the sense that even though the equipment is not personal to each user, an athlete participating in the sport has ownership of the barbell for an extended duration of time. It is also unique in the sense that a sensor can be embedded into a barbell and then its overall weight can be adjusted accordingly. It will not introduce any changes to the sporting regulations but make the barbell smart. A smart barbell would not only allow for improved training to track the bar position and speed, but also improve refereeing to judge an athlete's performance.

4.4.1 Theme 1: Improper Training Methods. The first theme that emerged from our data is improper training methods. A similar theme emerged in blind sports and it is unsurprising that it also emerged in this group. Participants from both blind sports expressed interest in need for accessible training aids to teach the basic skills required for the sport, as outlined in our earlier section.

Athletes are evaluated on their precision across several factors in para powerlifting. Currently, when athletes are in training camps, an expert coach views their form, judges their lift and helps them improve. However, the frequency of these training camps is low. Most of the times, the athletes are spread across the country and training remotely. They video record their lifts to show to their coach and solicit feedback. This reliance on remote video coaching is prone to error due to the limited view of the camera. The coaches are not able to provide adequate feedback at all times which may lead to failure in competitions. An additional issue with this method is athletes choose to send their 'best videos' instead of their failed attempts. The organization recently switched their process from videotaping to video conferencing to avoid this issue.

However, even if the remote coaching is conducted through video conferencing, it limits the number of athletes a coach can train at the same time. And within that training session, the limited view makes it hard to adequately assist an athlete reach their full potential. These issues point towards a need for a system that facilitates immediate feedback but also the ability to revisit an athlete's training session at a later time.

Para powerlifting athletes also expressed interest in gathering physiological data to improve their training performance. While several commercial wearables exist that may be able to measure and track this data, the athletes specifically wanted to instrument their benches instead of their body. 
"Okay. One of the things I was, you know, I was thinking about, and also I've been speaking with technological companies such as like Google and things of that nature about is developing some type of device that they could put insert on the bench to measure what muscles are being engaged in order to lift the weight at the correct speed, in order to generate the most power. [...] Well, I feel that if it's on the bench, it can be a little more universal. Um, and I think it would keep the costs a bit lower because I know that if it's a body mechanism, then you know, it might have to deal with like changing other pads and stuff like that. Or it might get in the way of the travel or it might feel uncomfortable um, when you're actually competing and lifting or even, you know, in training, you, um, lifting it. It might be uncomfortable to have, you know, a lot of wires and stuff connected to you." [A-3]

Lastly, participants in wheelchair tennis did not express a high interest in training aids, but this can be attributed to their already highly developed training programs.

4.4.2 Theme 2: Longitudinal Behavioral Tracking. The second major theme that emerged from our interviews in this group was the use of sensor equipped instruments to collect data that will facilitate aggregating longitudinal behavior of athletes and their performance. All participants viewed equipment instrumentation as a necessary means to the goal of tracking behaviors such as the physical and geometrical implications of not being able to use your legs to generate power in wheelchair tennis, or deviation in bar path based on the angle of the elbow in para powerlifting. The participants also stated that this data may be useful to convince others to reflect on their current practices. Para powerlifting coach wants to use longitudinal data to demonstrate the long-term implications of a certain technique whereas analysts in wheelchair tennis want to build a data-driven process to inform and improve the focus of training on certain aspects of the game. It has been seen in wheelchair tennis that most points are over within the first 3-4 shots. So, the wheelchair tennis analyst in our study explained:

"And I wasn't seeing on the practice court enough, in my mind, emphasis on serving return. Because if, if so, much of it is over uh, in zero to four, then why, why are we hitting up and down the court the whole time and working on that forehand? When actually most of the time you're just hitting serve return forehand or backhand and the points over. [...] just give them the data and then ask what implications this could have for the training court." [DA-1]

\section{OPPORTUNITIES}

Reflecting on the challenges encapsulated by our interviews, we highlight four opportunities to improve adaptive sports. We don't want to focus narrowly on analytics and instead use our work to urge HCI progress for collaboration, organizational management and manufacturing (new prototypes)- areas that should be the primary focus to improve the state of adaptive sports. These opportunities have the potential to have an immediate impact in improving the accessibility of adaptive sport and addressing their analytical needs.

\subsection{Tracking Wheelchair Health}

As stated earlier in section 4.3.2, the wheelchairs are expensive and they require regular maintenance. A clear opportunity is to instrument the wheelchair with sensors to monitor its health. A full profile of different parts of the wheelchair can help in tuning the chair setup but also ensure that parts may be replaced to prevent the wheelchair from sustaining irreparable damage. In fact, a continuous monitoring of the chair setup may even aid preventing possible injuries to athletes! This longitudinal tracking of chair setup can also help improve the initial wheelchair customization. The process that currently relies on an expert can be democratized using a data-driven process that could potentially leverage longitudinal data from athletes with different disabilities and injuries across several wheelchair-based sports. The availability of this data can also allow manufacturers to develop a standardized customization tests to determine optimal parameters for an athlete in the market for a new wheelchair. A similar approach is used in electronics manufacturing to test if the device fulfills its intended function [25].

\subsection{Smart Equipment-based Analytics}

Adaptive sports, especially wheelchair sports present a unique opportunity to instrument equipment like the wheelchair with sensors that would be able to gather data analytics for each athlete during the game. As seen in our findings, while some sports struggle with accessibility, there are some sports (e.g., wheelchair tennis) that seek to capture data analytics. A sensor on the spokes of the wheel can easily measure acceleration, top speed, turning radius, and more. Similarly, sports with high equipment such as para powerlifting can use sensors embedded inside a barbell to track the bar movement and bar velocity, two of the key performance metrics for which the coaches seek newer training methods (section 4.4.1). It points towards an immediate opportunity to build smart equipment to gather analytics for adaptive sports in a non-intrusive manner. It overcomes the reliance of adaptive sports on retrofitting video technology designed for able-bodied use for their own purposes.

In fact, data collected from sensors embedded on the wheelchair can be used to build large datasets and improve the underlying algorithm to gather analytics across different sports. It can overcome the issue of inaccessible analytic algorithms on fitness trackers.

\subsection{Smart Equipment as Training Aids}

Another direct opportunity that emerged from our themes was using smart equipment as training aids, especially to improve accessibility in blind sports. Additionally, blind sports need audio cues to even setup the game. So, the reliance on technology is higher than other adaptive sports. Blind hockey still suffers from poor robustness of the adaptive puck. Both blind hockey and beepball athletes noted specific examples of smart training equipment as well. For example, a hockey stick that makes particular noise when the stick completely touches the ice enables visually impaired athletes to learn hockey-dribbling skills. In beepball, a key difference in the adaptive sport is that the hitter needs to learn to swing at the exact location and the pitcher has to adjust. This learning process can be facilitated by a smart pitching machine that learns the natural swing angle for a hitter over time and adjusts accordingly. 
In fact, smart equipment can also be used to build improved communication tools. One participant [A-4] recounted his experience with blind skiing. They used a headset to communicate with a spotter that would guide them down the slope with navigation information. A similar idea was also expressed by the blind hockey coach [C-5]. They recounted that one of the junior players was ecstatic at the improved accessibility of the sport when they could hear their coach from a headset. While they acknowledged that this would not be allowed in competitive games, but it opens the door up for improving the user experience for a casual player, and perhaps as a training aid for a novice athlete.

We acknowledge that in certain cases, equipment instrumentation opportunities have not been overwhelmingly leveraged by able-bodied sports as well (e.g., a smart hockey stick), but such solutions may be imperative for the evolution of adaptive sports.

\subsection{Technology to Support Outreach and Recruitment}

A common challenge across all adaptive sports was their ability to recruit new players. This was partly due to financial and resource constraints, and partly due to lack of awareness about adaptive sports. Even the more well-known adaptive sports such as wheelchair basketball noted that they do not have an organized way to reach out to junior leagues. The high-performance manager [HPM-1] expressed interest in tools that could manage and register leagues at various levels and streamline the process for recruiting athletes for the national team. Such tools exist not only for sports, but in other domains as well. This is an opportunity for a tech company to quickly make a big impact on adaptive sports.

On the other hand, beepball faces difficulties with outreach and getting people interested in the sport. To bolster their outreach, some beepball teams started streaming their games using phones to broadcast to friends and family of people in the visually impaired community to generate excitement for the sport. However, [A-6] noted that it is hard for a visually impaired person to point and take good videos which makes it challenging to build engaging content. This challenge is not specific to adaptive sports but faced everyday by visually impaired individuals. Prior work has explored techniques to overcome the camera pointing issue [54]. It can also be combined with automatic synthesized audio description for a wider reach in the blind community [26]. There is an opportunity for researchers to leverage existing work in the accessibility domain and apply it for solving outreach challenges with blind sports.

\section{CONCLUSION}

We started this research by outlining how sensing and data analytics has positively impacted the evolution of sports. It has been possible due to proliferation in both research and commercial efforts to support the decision-making process in sports. However, we also discovered that the same opportunity was not afforded to adaptive sports. We learned that the problem lies not just with data analytics, but some adaptive sports are still struggling with issues of inaccessible technologies. As a result, adaptive sports have not gained popularity, the organizations are not able to recruit individuals or convince other organizations to invest in them. The financial and resource constraints feed back into the inaccessibility and outreach challenges making it a vicious cycle. As one may expect, our study highlighted that these challenges inhibit the growth of paraathletes. They cannot accurately track their physical conditioning, visualize their core performance metrics, and ultimately they are unable to make data-driven decisions. In our work we uncover issues that plague adaptive sports, highlight the challenges faced by different sports and outline opportunities where the community can make the most impact.

All our participants were passionate about their respective sport and have faced numerous challenges over the years. While some of these issues may seem obvious, in reality these drawbacks are simply creating inequality between adaptive and able-bodied sports. The themes that have emerged from our analysis point to opportunities that are immediately applicable and can make a big impact to improve the current state of adaptive sports. These opportunities span across multiple areas of sensing by instrumenting the equipment, making training aids more accessible, or enabling better content sharing for improved outreach. We urge researchers and technologists to partake in this research and development process to empower all stakeholders in adaptive sports, thereby uplifting the community as a whole.

\section{REFERENCES}

[1] [n.d.]. Smart Watches Could Do More For Wheelchair Users. https://fivethirtyeight.com/features/smart-watches-could-do-more-forwheelchair-users/. Accessed: 2020-09-17.

[2] Emad Abdelrasoul, Islam Mahmoud, Pro Stergiou, and Larry Katz. 2015. The accuracy of a real time sensor in an instrumented basketball. Procedia Engineering 112 (2015), 202-206.

[3] Marco Aggravi, Gionata Salvietti, and Domenico Prattichizzo. 2016. Haptic assistive bracelets for blind skier guidance. In Proceedings of the 7th Augmented Human International Conference 2016. 1-4.

[4] Ursina Arnet, Stefan van Drongelen, Matthias Schlüssel, Veronika Lay, Lucas HV van der Woude, and HEJ Veeger. 2014. The effect of crank position and backrest inclination on shoulder load and mechanical efficiency during handcycling. Scandinavian journal of medicine \& science in sports 24, 2 (2014), 386-394.

[5] Marc Bächlin, Kilian Förster, and Gerhard Tröster. 2009. SwimMaster: a wearable assistant for swimmer. In Proceedings of the 11th international conference on Ubiquitous computing. 215-224.

[6] Virginia Braun and Victoria Clarke. 2006. Using thematic analysis in psychology. Qualitative research in psychology 3, 2 (2006), 77-101.

[7] Patrick Carrington, Denzel Ketter, and Amy Hurst. 2017. Understanding fatigue and stamina management opportunities and challenges in wheelchair basketball. In Proceedings of the 19th International ACM SIGACCESS Conference on Computers and Accessibility. 130-139.

[8] Patrick Carrington, Gierad Laput, and Jeffrey P Bigham. 2018. Exploring the data tracking and sharing preferences of wheelchair athletes. In Proceedings of the 20th International ACM SIGACCESS Conference on Computers and Accessibility. 242-248.

[9] Paul Casella. 2015. Statcast primer: Baseball will never be the same. MLB. com (2015).

[10] Jr-Jung Chen, Yi-Fang Chung, Chiu-Ping Chang, Chung-Ta King, and ChengHsin Hsu. 2014. A wearable virtual coach for Marathon beginners. In 2014 20th IEEE International Conference on Parallel and Distributed Systems (ICPADS). IEEE, 915-920.

[11] Ed H Chi, Jin Song, and Greg Corbin. 2004. " Killer App" of wearable computing: wireless force sensing body protectors for martial arts. In Proceedings of the 17th annual ACM symposium on User interface software and technology. 277-285.

[12] Ashley Colley, Jani Väyrynen, and Jonna Häkkilä. 2015. Skiing in a blended virtuality: an in-the-wild experiment. In Proceedings of the 19th International Academic Mindtrek Conference. 89-91.

[13] Ahmet Ekin, A Murat Tekalp, and Rajiv Mehrotra. 2003. Automatic soccer video analysis and summarization. IEEE Transactions on Image processing 12, 7 (2003), 796-807.

[14] Anton Fedosov, Evangelos Niforatos, Ivan Elhart, Teseo Schneider, Dmitry Anisimov, and Marc Langheinrich. 2016. Design and evaluation of a wearable AR system for sharing personalized content on ski resort maps. In Proceedings of the 15th International Conference on Mobile and Ubiquitous Multimedia. 141-152. 
[15] Corinna Feeken, Merlin Wasmann, Wilko Heuten, Dag Ennenga, Heiko Müller, and Susanne Boll. 2016. ClimbingAssist: direct vibro-tactile feedback on climbing technique. In Proceedings of the 2016 ACM International foint Conference on Pervasive and Ubiquitous Computing: Adjunct. 57-60.

[16] Filipe Trocado Ferreira, Jaime S Cardoso, and Hélder P Oliveira. 2015. Video Analysis in Indoor Soccer using a Quadcopter.. In ICPRAM (1). 77-86.

[17] Eelke Folmer. 2015. Exploring the use of an aerial robot to guide blind runners. ACM SIGACCESS Accessibility and Computing 112 (2015), 3-7.

[18] Rob Gray. 2009. How do batters use visual, auditory, and tactile information about the success of a baseball swing? Research Quarterly for Exercise and Sport 80, 3 (2009), 491-501.

[19] Pål Halvorsen, Simen Sægrov, Asgeir Mortensen, David KC Kristensen, Alexander Eichhorn, Magnus Stenhaug, Stian Dahl, Håkon Kvale Stensland, Vamsidhar Reddy Gaddam, Carsten Griwodz, et al. 2013. Bagadus: an integrated system for arena sports analytics: a soccer case study. In Proceedings of the 4th ACM Multimedia Systems Conference. 48-59.

[20] Ping-Hsuan Han, Yang-Sheng Chen, Yilun Zhong, Han-Lei Wang, and Yi-Ping Hung. 2017. My Tai-Chi coaches: an augmented-learning tool for practicing TaiChi Chuan. In Proceedings of the 8th Augmented Human International Conference. $1-4$.

[21] Mahmoud Hassan, Florian Daiber, Frederik Wiehr, Felix Kosmalla, and Antonio Krüger. 2017. Footstriker: An EMS-based foot strike assistant for running. Proceedings of the ACM on Interactive, Mobile, Wearable and Ubiquitous Technologies 1,1 (2017), 1-18.

[22] Shivayogi V Hiremath, Dan Ding, and Rory A Cooper. 2013. Development and evaluation of a gyroscope-based wheel rotation monitor for manual wheelchair users. The journal of spinal cord medicine 36, 4 (2013), 347-356.

[23] Raine Kajastila and Perttu Hämäläinen. 2014. Augmented climbing: interacting with projected graphics on a climbing wall. In CHI'14 Extended Abstracts on Human Factors in Computing Systems. 1279-1284.

[24] Rushil Khurana, Karan Ahuja, Zac Yu, Jennifer Mankoff, Chris Harrison, and Mayank Goel. 2018. GymCam: Detecting, recognizing and tracking simultaneous exercises in unconstrained scenes. Proceedings of the ACM on Interactive, Mobile, Wearable and Ubiquitous Technologies 2, 4 (2018), 1-17.

[25] Rushil Khurana and Steve Hodges. 2020. Beyond the Prototype: Understanding the Challenge of Scaling Hardware Device Production. In Proceedings of the 2020 CHI Conference on Human Factors in Computing Systems. 1-11.

[26] Masatomo Kobayashi, Kentarou Fukuda, Hironobu Takagi, and Chieko Asakawa. 2009. Providing synthesized audio description for online videos. In Proceedings of the 11th international ACM SIGACCESS conference on Computers and accessibility. 249-250.

[27] Michinari Kono, Takashi Miyaki, and Jun Rekimoto. 2017. JackIn airsoft: localization and view sharing for strategic sports. In Proceedings of the 23rd ACM Symposium on Virtual Reality Software and Technology. 1-4.

[28] Anton Kos and Anton Umek. 2018. Smart sport equipment: SmartSki prototype for biofeedback applications in skiing. Personal and Ubiquitous Computing 22, 3 (2018), 535-544.

[29] Marcos Lage, Jorge Piazentin Ono, Daniel Cervone, Justin Chiang, Carlos Dietrich, and Claudio T Silva. 2016. Statcast dashboard: Exploration of spatiotemporal baseball data. IEEE computer graphics and applications 36, 5 (2016), 28-37.

[30] Michael Lapinski, Eric Berkson, Thomas Gill, Mike Reinold, and Joseph A Paradiso. 2009. A distributed wearable, wireless sensor system for evaluating professional baseball pitchers and batters. In 2009 International Symposium on Wearable Computers. IEEE, 131-138.

[31] Amy Lastuka and Michael Cottingham. 2016. The effect of adaptive sports on employment among people with disabilities. Disability and rehabilitation 38,8 (2016), 742-748.

[32] Neil R Lundberg, Stacy Taniguchi, Bryan P McCormick, and Catherine Tibbs. 2011. Identity negotiating: Redefining stigmatized identities through adaptive sports and recreation participation among individuals with a disability. fournal of Leisure Research 43, 2 (2011), 205-225.

[33] Fabricio Anicio de Magalhaes, Giuseppe Vannozzi, Giorgio Gatta, and Silvia Fantozzi. 2015. Wearable inertial sensors in swimming motion analysis: a systematic review. Fournal of sports sciences 33, 7 (2015), 732-745.

[34] Jonathan C Maglott, Junkai Xu, and Peter B Shull. 2017. Differences in arm motion timing characteristics for basketball free throw and jump shooting via a body-worn sensorized sleeve. In 2017 IEEE 14th International Conference on Wearable and Implantable Body Sensor Networks (BSN). IEEE, 31-34.

[35] Barry Mason, John Lenton, James Rhodes, Rory Cooper, and Victoria GooseyTolfrey. 2014. Comparing the activity profiles of wheelchair rugby using a miniaturised data logger and radio-frequency tracking system. BioMed research international 2014 (2014).

[36] Dan Morris, T Scott Saponas, Andrew Guillory, and Ilya Kelner. 2014. RecoFit: using a wearable sensor to find, recognize, and count repetitive exercises. In Proceedings of the SIGCHI Conference on Human Factors in Computing Systems. 3225-3234.

[37] Annika Muehlbradt, Varsha Koushik, and Shaun K Kane. 2017. Goby: A wearable swimming aid for blind athletes. In Proceedings of the 19th International ACM SIGACCESS Conference on Computers and Accessibility. 377-378.

[38] Le Nguyen Ngu Nguyen, Daniel Rodríguez-Martín, Andreu Català, Carlos PérezLópez, Albert Samà, and Andrea Cavallaro. 2015. Basketball activity recognition using wearable inertial measurement units. In Proceedings of the XVI international conference on Human Computer Interaction. 1-6.

[39] NEIL Owens, C Harris, and C Stennett. 2003. Hawk-eye tennis system. In 2003 International Conference on Visual Information Engineering VIE 2003. IET, 182-185.

[40] Ashutosh Raina, TG Lakshmi, and Sahana Murthy. 2017. CoMBaT: Wearable technology based training system for novice badminton players. In 2017 IEEE 17th International Conference on Advanced Learning Technologies (ICALT). IEEE, 153-157.

[41] Kyle Rector, Cynthia L Bennett, and Julie A Kientz. 2013. Eyes-free yoga: an exergame using depth cameras for blind \& low vision exercise. In Proceedings of the 15th International ACM SIGACCESS Conference on Computers and Accessibility. 1-8.

[42] K Andrew R Richards and Michael A Hemphill. 2018. A practical guide to collaborative qualitative data analysis. Fournal of Teaching in Physical Education 37, 2 (2018), 225-231.

[43] Minna Ruckenstein. 2014. Visualized and interacted life: Personal analytics and engagements with data doubles. Societies 4, 1 (2014), 68-84

[44] Ajay K Sarkar, Daniel A James, Andrew W Busch, and David V Thiel. 2011. Triaxial accelerometer sensor trials for bat swing interpretation in cricket. Procedia Engineering 13 (2011), 232-237.

[45] Matthias Seuter, Lucien Opitz, Gernot Bauer, and David Hochmann. 2016. Livefeedback from the imus: Animated 3d visualization for everyday-exercising. In Proceedings of the 2016 ACM International foint Conference on Pervasive and Ubiquitous Computing: Adjunct. 904-907.

[46] Long Sha, Patrick Lucey, Yisong Yue, Xinyu Wei, Jennifer Hobbs, Charlie Rohlf, and Sridha Sridharan. 2018. Interactive sports analytics: An intelligent interface for utilizing trajectories for interactive sports play retrieval and analytics. ACM Transactions on Computer-Human Interaction (TOCHI) 25, 2 (2018), 1-32.

[47] Daniel Spelmezan. 2012. An investigation into the use of tactile instructions in snowboarding. In Proceedings of the 14th international conference on Humancomputer interaction with mobile devices and services. 417-426.

[48] Michelle L Sporner, Garrett G Grindle, Annmarie Kelleher, Emily E Teodorski, Rosemarie Cooper, and Rory A Cooper. 2009. Quantification of activity during wheelchair basketball and rugby at the National Veterans Wheelchair Games: A pilot study. Prosthetics and orthotics international 33, 3 (2009), 210-217.

[49] Craig D Stewart, Penny Traitor, and Vicki L Hanson. 2014. I'd Tap That! providing real time feedback on roller derby skills. In CHI'14 Extended Abstracts on Human Factors in Computing Systems. 2221-2226.

[50] Michelle Lynn Tolerico. 2005. Investigation of the mobility characteristics and activity levels of manual wheelchair users in two real world environments. Ph.D. Dissertation. University of Pittsburgh.

[51] Martin Tomitsch, Wolfgang Aigner, and Thomas Grechenig. 2007. A concept to support seamless spectator participation in sports events based on wearable motion sensors. In 2007 2nd International Conference on Pervasive Computing and Applications. IEEE, 209-214.

[52] RMA Van Der Slikke, MAM Berger, DJJ Bregman, AH Lagerberg, and HEJ Veeger. 2015. Opportunities for measuring wheelchair kinematics in match settings; reliability of a three inertial sensor configuration. Fournal of Biomechanics 48, 12 (2015), 3398-3405.

[53] Stefan van Drongelen, Jos van den Berg, Ursina Arnet, DirkJan HEJ Veeger, and Lucas HV van der Woude. 2011. Development and validity of an instrumented handbike: initial results of propulsion kinetics. Medical engineering \& physics 33, 9 (2011), 1167-1173.

[54] Marynel Vázquez and Aaron Steinfeld. 2012. Helping visually impaired users properly aim a camera. In Proceedings of the 14th international ACM SIGACCESS conference on Computers and accessibility. 95-102.

[55] Zhelong Wang, Ming Guo, and Cong Zhao. 2016. Badminton stroke recognition based on body sensor networks. IEEE Transactions on Human-Machine Systems 46, 5 (2016), 769-775. 